\title{
Relative distance of lights: An extension of Bugelski's findings
}

\author{
BRUCE E. DUNN 1 \\ UNIVERSITY OF CALGARY
}

Three lights in various orientations were presented to two trained Ss. The Ss adjusted the three lights until they appeared equidistant. The results supported earlier work of a similar nature, in that the higher light was perceived as closer and horizontal separation between lights produced no significant differences in depth settings. The phenomenon was shown to occur with monocular vision, fixated vision, large angular separation of the stimuli, and with different directions of regard with respect to the fixation point. The relationship to other research is discussed.

Roelofs and Zeeman (1957) showed that a stimulus presented to the nasal part of the eye was seen as closer to the $S$ than the stimuli presented to the temporal part of the eye. Dunn, Gray, and Thompson (1965) showed that the height of an object in the visual field was a critical variable in its perceived distance relative to another object in the field. This result is also recorded by Roelofs and Zeeman (1957) and by Epstein (1966). The most usual finding has been that the higher of two stimuli is seen as farther away. In none of these cases has there been control for position of the S's eye in the field. Dunn (1967) attempted to vary the direction of regard, but was unable to find any differences. However, he used transparencies that varied in their orientation to the $S$ as a function of the direction from which the $S$ looked at them. The findings in all cases supported the earlier studies. The higher end of the transparency was perceived as the farther end. Bugelski (1967) obtained results that differed from the above studies. He used two lights, separated by $2 \mathrm{deg}$, in an otherwise dark field. The $S$ adjusted the lights to make them appear equal in distance. All but two Ss adjusted the lights so that the higher of the two lights was placed closer. Under another condition, two stimuli were presented to the Ss on the same horizontal, also separated by 2 deg. There was no significant statistical difference between the mean placements of the two lights. Bugelski postulated that the vertical effect would probably not occur with separations of much over 2 deg.

The disparity between the above findings raises several interesting questions. First, did the free movement of the S's head allowed by Bugelski enter into the results? Second, was the direction of regard with respect to the stimulus display a factor? Third, was the small visual angle a factor, as suggested by Bugelski? Fourth, was the fact that binocular vision was used by the Ss a significant factor? The following study attempts to answer the questions raised above. The S's head was fixed with a chin-rest in order to avoid head movement. He fixated upon a center fixation light to direct his direction of regard. Vision was monocular and the angle between the stimuli was much larger than that used by Bugelski. The apparatus was so constructed that direction of regard could be varied while maintaining rigid relationships between important aspects of the stimulus amay.

\section{Subjects}

\section{METHOD}

The Ss were two sophisticated male and female undergraduate psychology majors. Their training consisted of several months of making judgments in various relative depth situations, not associated with the apparatus used in the following experiment. Both Ss had considerable experience in maintaining fixation upon a fixation light. Neither $S$ was familiar with Bugelski's findings.

\section{Apparatus}

The $\mathrm{S}$ viewed the stimuli through a $1-\mathrm{cm}$ pupil from a distance of $1 \mathrm{~cm}$. His chin rested on a chin-rest that held his head motionless. Three 1/2-in. pipes, painted on the inside with low-reflectant black paint, were oriented so that they were at different angles to the plane of the cornea. Lines projected from the pipes would have converged at a point slightly behind the pupil of S's eye. Within each pipe was placed a small circular stimulus, $1 / 2$ in. in diam, painted with luminous paint. The stimuli could be move toward and away from $S$ by three reversible motors under the control of both $E$ and $S$. The intensity of the light of the three luminous stimuli was controlled by illumination between trials. The amount of illumination was calibrated individually for the three stimuli. The pipes were hinged to a rotating plate in which the eyepiece was fitted. By means of the hinges and the plate, the angle at which the stimuli approached the eye could be changed as could the angle of the plane, formed by the three pipes, to the ground. In the present experiment, each pipe was at an angle of $20 \mathrm{deg}$ to the adjacent pipe. Thus, no matter how the $S$ moved the stimuli, the visual angle of their separation remained a constant. The angle of the plane, formed by the pipes, to the ground was either horizontal or vertical.

\section{Procedure}

Each of the two trained Ss received a total of 80 trials, during which he adjusted the left-most and right-most or upper-most and lower-most stimuli to appear equal in distance to each other and to a center stimulus randomly preset to a distance of between 31 and $99 \mathrm{~cm}$ from his eye (S was permitted to move the center stimulus if he was unable to make a match). Eight different angular relationships between the stimuli were used. In all cases, the angle between the two extreme pipes was $40 \mathrm{deg}$, while the center pipe containing the fixation stimulus was midway between. Under conditions where the plane of the pipes was horizontal, the fixation stimulus was placed in the following five positions with respect to the plane of the cornea: $0 \mathrm{deg}, 10 \mathrm{deg}$ temporally, $10 \mathrm{deg}$ nasally, $20 \mathrm{deg}$ temporally, and $20 \mathrm{deg}$ nasally. Under those conditions, in which the plane of the pipes was vertical, three different center stimulus placements were used: $0 \mathrm{deg}, 10 \mathrm{deg}$ above the eye level, and $10 \mathrm{deg}$ below the eye level of S.

Each $S$ received 10 presentations of each stimulus condition. Each $S$ was run for 8 days. On any given day, he received 10 stimulus presentations, 5 at each of two conditions. The order of presentation of the conditions was randomized over days for each $\mathrm{S}$. The Ss were light adapted for $5 \mathrm{~min}$ between each presentation to prevent their being able to detect details of the light reflected from the luminous discs. A day or session lasted about $11 / 4 \mathrm{~h}$.

Possible differences between the two external stimuli were controlled by rotating the apparatus 180 deg so that the positions of the two external stimuli were completely reversed. The center stimulus that did not enter into the calculation of the results was always the same stimulus disc. 
Table 1

Average Log Relative Depth of Lights (Values are obtained by subtraction. See text for explanation.)

Pipes on Horizontal Plane

Position of

Fixation Light

\begin{tabular}{lccc}
\hline $0 \mathrm{deg}$ & .0096 & $0 \mathrm{deg}$ & .0578 \\
$10 \mathrm{deg}$ nasal & -.0066 & $10 \mathrm{deg}$ down & .0326 \\
$10 \mathrm{deg}$ temporal & .0118 & $10 \mathrm{deg}$ up & .0220 \\
20 deg nasal & .0084 & & \\
20 deg temporal & .0044 & & \\
\hline
\end{tabular}

\section{RESULTS}

The Ss' judgments were converted into logarithms. Under the horizontal conditions, the perceived distance of the stimulus striking the nasal part of the eye was subtracted from the $\log$ of the distance of the stimulus striking the temporal part of the eye. In the vertical condition, the $\log$ of the distance of the higher stimulus was subtracted from the $\log$ of the distance of the lower stimulus. The results of these operations are shown in Table 1. Note that the effects under the horizontal conditions are minimal and not very consistent. However, the effects under the vertical condition are much larger and, although they differ under the different directions of regard, all results are in the same direction, namely that the higher stimulus is placed closer to $S$.

An F-Max test showed there to be no significant heterogeneity of variance, so an analysis of variance was performed on the data. The cut-off of $p<.05$ was selected as the criterion of significance. Under horizontal conditions, the only significant effect was the control condition which consisted of rotating the whole apparatus $180 \mathrm{deg}$. An F $=124.16(\mathrm{p}<.05)$ was obtained for the analyses of this variable. Under the vertical condition, three variables showed significant effects. The analysis of the height of the stimulus gave an F of $58.97(\mathrm{p}<.05)$, the variable of viewing angle or direction of regard with 2 deg of freedom gave an $F$ of $5.00(p<.05)$, while again the control variable was significant, giving an $F$ of $75.90(p<.05)$. Under none of these conditions was the $S$ variable significant, nor were any of its interactions. Also, under both vertical and horizontal conditions, Ss were considered a fixed variable since there was no strictly definable population from which the $S s$ could be said to be randomly sampled.

\section{DISCUSSION}

The results answer rather concisely the four questions asked in the introduction. Bugglski's results can be obtained when head and even eye movement is not permitted. His results are obtainable under all conditions of fixation used in this experiment. The results are also obtainable under conditions of much wider separation of the stimuli than he used. Binocular vision is not necessary. As in Bugelski's experiment, the position of the horizontal stimuli gave no significant difference in the settings, despite a wider range of direction of regard for the horizontal plane than was used for stimuli in the vertical plane. Finally, the illusion is compelling enough to clearly affect Ss trained in making relative distance judgments.

The results of this study, while supporting those of Bugelski, do not resolve the conflict between the results discussed in the introduction. The significant difference between the directions of regard found in the study using vertically placed stimuli does not help in this explanation. Had the results been such that the higher the stimulus array was with respect to the S's eye, the greater distance the higher stimulus appeared, the results would have been consistent with the basic geometry of the every-day visual field in which stimuli over the head of the $S$ reverse their information with respect to height in the visual field. That is, for high stimuli, the further-away stimulus looks lower, while for the low stimuli, the further-away stimulus hooks higher (see Dunn et al, 1965). The results of this study appear to indicate that the effect is greater when the $S$ is fixated straight ahead at a point that might be viewed as the equivalent of the horizon point in a filled field of view. A Duncan Range Test shows that the only condition that is different among the three is that condition in which the $S$ is looking straight ahead. One is tempted to postulate something about differences in the retinal-image size of equal-size stimuli focuses in the superior and inferior hemisphere of the: eye, but closeness of the stimuli and the potential for head movement in Bugelski's study argue against this. It is certainly true, however, that perceptually the higher of two stimuli actually of equal size and distance must appear to be a smaller object if it appears nearer. This finding then is quite consonant with the moon-illusion phenomenon. Perhaps the difference in the effect is due to muscular involvement of the 10-deg change in fixation done by eye as opposed to head movement. If this were the case, then a ceiling-plane set on the part of the Ss to three "floating" lights could be postulated to explain the discrepancy between the earlier findings on the effect of height in the visual field on perceived relative depth and the findings in the Bugelski study and this study. Thus, if $S$ perceives both stimuli or all of a single stimulus to be on a plane below his eye level, the higher stimulus, if there are two, or the higher part of a single stimulus should look farther. If he perceives the stimuli or stimulus to be on a plane above his eye level (ceiling-plane), the opposite result should occur.

\section{REFERENCES}

BUGELSKI, B. R. Traffic signals and depth perception. Science, 1967, $157,1464-1465$.

DUNN, B. E., GRAY, G. C., \& THOMPSON, D. Relative height on the picture-plane and depth perception. Perceptual \& Motor Skills, 1965, $21,227-236$

EPSTEIN, W. Perceived depth as a function of relative height under three background conditions. Joumal of Experimental Psychology, 1966, 72, 335-338.

ROELOFS, C. O., \& ZEEMAN, W. P. C. Apparent size and distance in binocular and monocular distance. Opthalmologica, 1957, 133, 188-204.

\section{NOTE}

1. Address: Psychology Department, University of Calgary, Calgary 44, Alberta, Canada.

(Accepted for publication April 28, 1969.) 